\title{
Analysis of choice in honeybees
}

\author{
P. A. COUVILLON and M. E. BITTERMAN \\ University of Hawaii, Honolulu, Hawaii
}

\begin{abstract}
Free-flying honeybees were trained in a set of four problems to choose between two differently scented targets, one or the other of which contained sucrose solution. The training was simulated quantitatively, always with the same simple linear equations for computing changes produced by reinforcement and nonreinforcement in the strength of association between each target and the sucrose, but with a diverse array of functions for predicting choice on the basis of relative strength. Accuracy of prediction was indexed by the root-mean-square (RMS) deviation of simulated data from real data. The results provide some good approximations of what is tentatively assumed to be the true choice function, setting the stage for further development of the associative features of the model to encompass more complex phenomena of honeybee learning in choice situations.
\end{abstract}

The purpose of this work, which continues our analysis of learning in honeybees from the perspective of learning in vertebrates (Couvillon \& Bitterman, 1980), was to analyze the process of choice. We are interested in choice because it provides by far the best measure of learning in free-flying honeybees. Latency of response to a single target, at least in one-trial-per-visit problems, is not very reliable, because the zero point (first appearance of the subject on its return from the hive) is difficult to fix; furthermore, there is a good deal of variation in readiness to land that seems unrelated to the attractiveness of the target. Resistance to extinction (persistent contact with an unbaited target), which we have used with some success in the past, is relatively inefficient. For example, to plot the course of acquisition in terms of resistance to extinction, multiple groups are required-a separate group for each degree of training-whereas the choice measure requires only a single group. The choice measure may seem unduly complex, in that it reflects the associative properties of two or more targets (between or among which the choice is made) rather than those of a single target, and it must reflect as well the operation of some rule of choice, but the relative simplicity of singletarget measures in these respects is deceptive. The single target is in reality a cluster of sensory components that interact with each other in complex ways of which we remain ignorant until we begin to study differential responding, with either simultaneous or separate presentations of different targets (Couvillon \& Bitterman, 1982; Couvillon, Klosterhalfen, \& Bitterman, 1983). Furthermore, although it may be possible in work with a single target to treat response as a simple reflection of associa-

This research was supported by Grant BNS 83-17051 from the National Science Foundation. Helpful advice from William T. Woodard on the interpretation of the data is gratefully acknowledged. Requests for reprints should be addressed to the Békésy Laboratory of Neurobiology, 1993 East-West Road, Honolulu, HI 96822. tive strength, it is necessary sooner or later to distinguish between associative processes and those responsible for the translation of association into performance. An advantage of the choice procedure is that it keeps us in contact with the problem of performance from the outset, and our efforts here were in fact focused on discovering the rule of choice.

Our method was to train individual foragers to fly from the hive to the sill of an open laboratory window, on which two targets discriminably different in odor were presented. One of the targets contained a drop of tap water (unacceptable to the bees), and the second contained a drop of sucrose solution from which feeding to repletion was permitted. In the simplest case (100:0), the location of the sucrose was perfectly predictable, but in other cases the location was predictable only imperfectly (as in the $75: 25$ case, in which the sucrose was three times as likely to be found on one target as on the other) or not at all $(50: 50)$. If, on any visit, an animal went first to the target containing water, it was free at once to correct its choice. The significance of the correction procedure for the present purposes is that the distribution of reinforcements is completely controlled, although it should be noted that there is no other practical way to work with free-flying subjects, which, if not fed on a given visit to the laboratory, forage elsewhere and may fail to return for hours.

In analogous experiments with vertebrates of different classes, several rather distinctive modes of adjustment have been observed, and the possibility has been suggested that they reflect the operation of different choice mechanisms (Bitterman, 1975). One familiar outcome is matching, a tendency for the choice ratio to equal the reinforcement ratio, and another is maximizing, a tendency toward exclusive choice of the higher-probability alternative. A contrary suggestion, based on the fact that the performance observed in these experiments often is not easily categorized (there are many degrees of overmatching, or undermaximizing, and even of undermatching), is that matching and maximizing are simply two points on a be- 
havioral continuum governed by a common maximizing mechanism (Sutherland \& Mackintosh, 1971). According to this view, differences in performance are due to differences in ability to maintain attention to relevant stimuli in the face of inconsistent reinforcement (Mackintosh, 1974, p. 172). Little is known about the behavior of invertebrates in such experiments. Matching has been found in cockroaches (Nauphoeta cincera) trained to avoid shock by turning left or right in a maze (Longo, 1964). Sigurdson's (1981) demonstration in our laboratory of matching in temporarily confined honeybees trained with small rewards and massed trials was the immediate precursor of the present experiments, which were carried out under the conditions more commonly used in our work.

The choice behavior of vertebrates (mainly pigeons) has been studied also in free-operant experiments (mainly with concurrent interval schedules). A common finding in that work has been a striking correspondence between the distribution of responses and the distribution of obtained reinforcements (Herrnstein, 1970), a phenomenon referred to as matching, which should not be confused with the matching often shown by pigeons in discrete-trials experiments (cf. Bullock \& Bitterman, 1962); as Mackintosh (1974) has noted, even an animal that responds exclusively to a higher-probability alternative, and therefore obtains all its reinforcements for response to that alternative, is matching in the free-operant definition of the term. There is as little agreement about the meaning of the free-operant results as about the meaning of the discrete-trials results (Herrnstein, 1982), but what seems clear, in any case, is that free-operant methods are not the best methods with which to begin the study of choice: They do not provide adequate control of the distribution of reinforcéments, which depends on the animal's behavior, or of the probabilities of reinforcement at moments of choice, which vary widely in the course of each session (Shimp, 1982).

The highly controlled discrete-trials method that we preferred to use here has been criticized as unnatural (Kamil, Peters, \& Lindstrom, 1982)-too unnatural, at least, to have any relevance to the problem of foraging which has been attracting so much attention of late-and it may be well to note our rejection of that criticism, although our primary concern is not to understand foraging. We find little reason to believe that the functional properties of an animal are discoverable only in experimental situations designed to mimic field conditions, or that they are so specific as to have no application outside the situations in which they are discovered (Menzel \& Bitterman, 1983). With respect to the problem of foraging, it may be well to note also that our interest here is not in what might be best for an animal to do under given circumstances, but in what the animal actually does and what that tells us about the sort of animal it is-about how its attraction to stimuli changes with reinforcement and nonreinforcement, and about how it makes choices.

\section{METHOD}

\section{Subjects}

The subjects were honeybees (Apis mellifera) that were foraging for nectar. They came from our own hives, situated in the vicinity of the laboratory.

\section{Procedure}

Individual bees were pretrained to fly from their hives to the laboratory and drink to repletion from a large drop (about $100 \mu \mathrm{l}$ ) of 50\% sucrose solution on a single target that was set on the sill of an open window. An animal was selected at random from a group of foragers at a feeding platform equipped with a large jar of $10 \%-15 \%$ sucrose solution, carried in a small matchbox to the laboratory, and placed on the target; it was permitted to drink its fill of the $50 \%$ solution (during which time it was marked with a spot of colored lacquer) and then to fly to the hive. Typically, the animal returned to the laboratory of its own accord within 3 to $5 \mathrm{~min}$ and continued thereafter to shuttle back and forth between the hive and the laboratory as long as food was available. If the marked bee did not return to the laboratory after the first placement, it was picked up again at the feeding platform (where it usually could be found), carried back to the laboratory in the matchbox, and set down on the target once more. The pretraining ended with the bee's second return to the laboratory of its own accord.

The targets were covered petri dishes of clear plastic, $5.5 \mathrm{~cm}$ in diameter. In each cover, which was sprayed with flat gray paint, eight equally spaced holes, $.5 \mathrm{~cm}$ in diameter, were drilled at the outer circumference. Two sets of targets were used in the training. The dishes of one set contained wads of cotton that were impregnated with a jasmine scent, and the dishes of the second set were scented in the same way with lemon (neither of these scents occurred in the field). The covers used on each visit were washed and exchanged for others in the same set after the visit in order to balance extraneous stimuli. For purposes of pretraining only, there was a third set of targets, labeled with both scents.

On each training visit, the animal found two targets set $10 \mathrm{~cm}$ apart on a line parallel to the outer edge of the window sill. One of the targets was scented with jasmine, the other with lemon, and the positions of the two targets were balanced in quasi-random fashion over visits. One of the targets contained a large drop of $50 \%$ sucrose solution, and the other a large drop of tap water that was distinguishable from the sucrose only by taste. The target first chosen by the subject on each visit was recorded. At the conclusion of the scheduled series of visits, the subject was captured and destroyed.

100:0 $\rightarrow 75: 25$ training. In the first stage of this experiment, 8 subjects were trained with one of the odors consistently reinforced and the other never, to the criterion of four successive correct choices. For half the animals, jasmine was reinforced, and for the rest, lemon. Upon reaching criterion, each animal was shifted for 32 additional visits to a 75:25 problem in which the odor that before had been consistently reinforced now was reinforced on only 3 visits in each block of 4 . Two different 75:25 schedules were used, one for 4 subjects and the other for the rest. Throughout the training, position was balanced in quasi-random fashion, with each of the odors reinforced as often on the right as on the left.

50:50 training. Each of 9 bees was permitted 20 visits to the two odors, with each odor reinforced equally often in each of the two positions. A single 50:50 schedule was used, but odor was balanced in the sense that the odor reinforced on the first visit was jasmine for five bees and lemon for the rest. The odor reinforced on the first visit was reinforced also on visits $4,5,8,10,12,14$, 16,17 , and 18 .

80:20 training. Each of 10 bees was permitted 16 visits to the two odors. One of the odors (jasmine for half the animals and lemon 
for the rest) was reinforced on visits 1,6 , and 11 , and the other odor was reinforced on the remaining visits, with position balanced over two subgroups of 5 animals each. This is not, of course, a conventional 80:20 problem, but the small number of training trials (warranted by the rapidity of associative change) also is unconventional by vertebrate standards; the regularity of the schedule permitted us to look for variation in the effects of reinforcement and nonreinforcement with continued training.

100:0 $\rightarrow 0: 100$ training. Each of 8 bees was permitted 20 visits to the two odors, one of which was consistently reinforced on visits 1-10 and the other on visits 11-20. Half the animals began with jasmine reinforced and the rest with lemon reinforced. Each odor was reinforced equally often in each position.

\section{RESULTS}

In the first stage of the $100: 0 \rightarrow 75: 25$ training, acquistion was rapid. The mean number of visits to criterion, including the criterion visits, was 6.25 (range, 4-8). Performance in the subsequent 75:25 training is plotted in Figure 1 in terms of the mean proportion of choices of A (defined here as the .75 alternative) in each block of 4 visits. For all 32 visits combined, the mean number of A choices was $24(\mathrm{SE}=1.02)$, exactly the matching value, but that was no longer very interesting, because inspection of the performance of each of the two small subgroups suggested the feasibility of a more refined analysis. At the outset of this work, we still were thinking in terms of the vertebrate results, looking for relations between asymptotic choice ratios and reinforcement ratios in blocks of visits, however small. Now we realized, as we might have realized before, that the effect of a single experience with reward or nonreward was large enough under the conditions employed to permit a visit-by-visit analysis. Accordingly, the schedule of reinforcement in each subsequent problem was kept the same for each subject in the group rather than being balanced over subgroups.

In Figure 2, performance in the 50:50 training is plotted in terms of the proportion of animals choosing alterna-

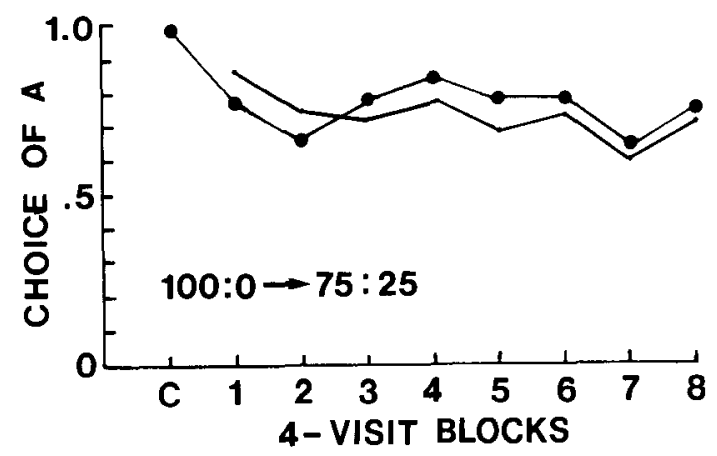

Figure 1. Mean proportion of $A$ choices $(A=$ the $\mathbf{. 7 5}$ alternative) in the criterion 100:0 block of four visits $(C)$ and in eight subsequent blocks after the reinforcement ratio was changed to 75:25 (large filled circles). The accompanying plot (small points) shows the results of a simulation with the choice function $K=.75, \mathrm{~s}=.625$, initial $V_{\mathrm{A}}=$ $\mathrm{V}_{\mathrm{B}}=.3$, and $\mathrm{U} \beta=\mathrm{D} \beta=.25$.

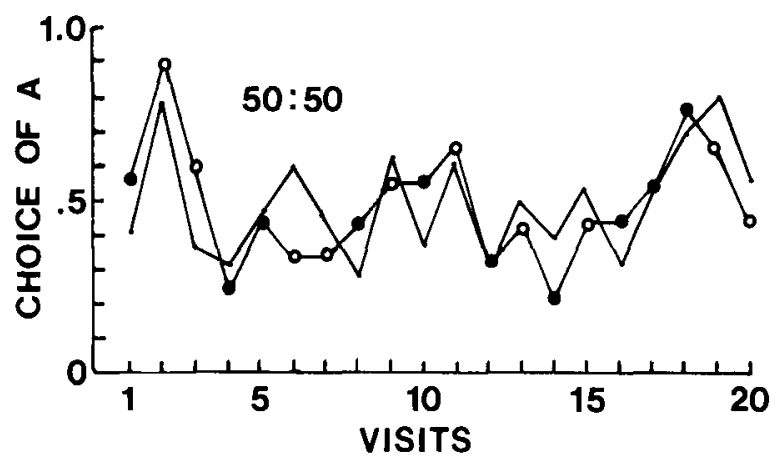

Figure 2. Proportion of $A$ choices $(A=$ the odor reinforced on the first visit) on each visit in the 50:50 training; the large filled circles are for visits on which $A$ was reinforced and the large open circles are for visits on which $B$ was reinforced. The accompanying plot (small points) shows the results of a simulation with the choice function $K=.75, s=.625$, initial $V_{A}=V_{B}=.3$, and $U \beta=D \beta=$ .25 .

tive $A$ on each visit ( $A$ is defined here as the odor reinforced on the first visit). The data points for visits on which $\mathrm{A}$ was reinforced are shown by filled circles; the points for visits on which the other odor, B, was reinforced, are shown by unfilled circles. The mean number of A choices in the 20 visits was $9.9(\mathrm{SE}=.83)$, but the curve is of interest primarily because of the relation between the pattern of change and the schedule of reinforcement, which expresses a modest but significant tendency for the proportion of animals choosing $A$ to increase after $\mathrm{A}+$ visits and to decrease after $\mathrm{B}+$ visits. The mean number of visits on which the animals chose the odor reinforced on the immediately preceding visit was 11.7 $(\mathrm{SE}=.47$ ), compared with the chance expectancy of 9.5 based on 19 visits $[\mathrm{t}(8)=4.68, \mathrm{p}<.01]$. It should be noted that there was no such influence of position, which seemingly was not very salient; the mean number of visits on which the animals chose the position reinforced on the immediately preceding visit was $9.3(\mathrm{SE}=1.7)$, compared with the chance value of $9.5(\mathrm{t}<1)$.

In Figure 3, performance in the 80:20 training is plotted in terms of the proportion of animals choosing A (defined here as the more frequently reinforced alternative) on each visit. The data points for visits on which $A$ was reinforced are shown by filled circles and the points for $B$ are shown by open circles. After some wide initial fluctuation, the curve settles at a level close to the matching value, an outcome that is not an artifact of averaging the scores of a majority of animals that were maximizing with those of a few poor performers. In the last 10 visits, every animal chose $B$ at least once- 2 animals four times, 4 animals three times, 2 animals two times, and 2 animals once (mean A choice $=7.4, \mathrm{SE}=.34$ )-although in 100:0 training with the same stimuli the probability of choosing the consistently reinforced alternative rapidly approaches 1 . The results suggest that the occasional visits on which $B$, rather than $A$, was reinforced continued to 


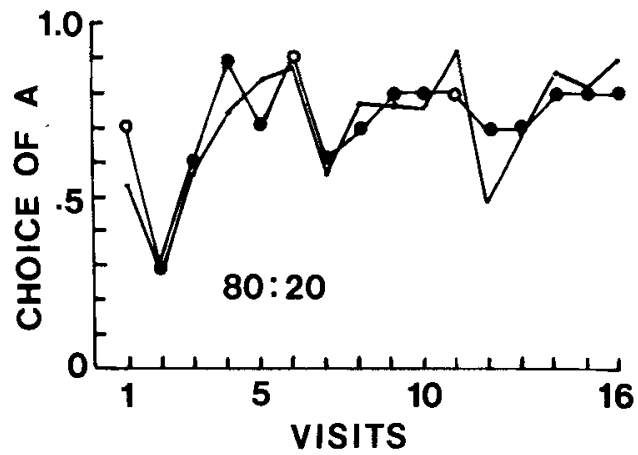

Figure 3. Proportion of $A$ choices $(A=$ the more frequently reinforced alternative) on each visit in the 80:20 training; the large filled circles are for visits on which $A$ was reinforced and the large open circles for visits on which B was reinforced. The accompanying plot (small points) shows the results of a simulation with the choice function $K=.75, s=.625$, initial $V_{A}=V_{B}=.3$, and $U \beta=D \beta=$ .25 .

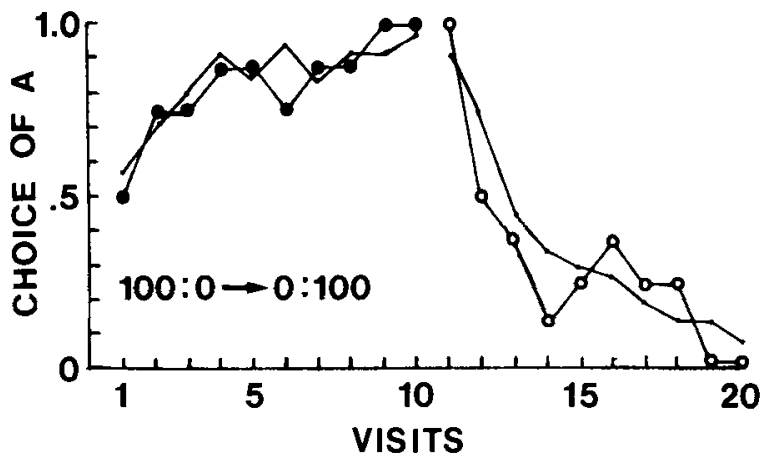

Figure 4. Proportion of $A$ choices $(A=$ the originally reinforced alternative) on each visit in the 100:0 $\rightarrow 0: 100$ training; the large filled circles are for visits on which $A$ was reinforced and the large open circles for visits on which B was reinforced. The accompanying plot (small points) shows the results of a simulation with the choice function $K=.75, s=.625$, initial $V_{A}=V_{B}=.3$, and $U \beta=D \beta=$ .25 .

influence the choice of the animals; as Figure 3 shows, each of the three $B+$ visits was followed by a decline in the proportion of animals choosing $\mathrm{A}$.

In Figure 4, performance in the 100:0 $\rightarrow 0: 100$ training is plotted in terms of the proportion of animals choosing A (defined here as the originally reinforced alternative) on each visit. The curves show rapid acquisition followed by rapid reversal. All animals chose A on the last two acquisition visits and on the first reversal visit; none chose A on either of the last two visits of the reversal training.

\section{INTERPRETATION}

Our approach to these data reflects the primacy of our interest in learning, that is, in the way in which experience changes the properties of the alternatives between which the animal must choose. We are interested in choice only secondarily, as an expression of that change. The choice rule we are looking for is one that relates probability of choice not to experience directly, but to the properties of the alternatives-which themselves are changed by experience in accordance with rules that must be specified if the choice rule is to be discovered. Our hypotheses about choice cannot be evaluated independently of hypotheses about learning. Because the learning experiments that provided our data were simple, we begin with simple learning rules-which undoubtedly will have to be extended to deal with the data of more complicated experiments, although it is not inconceivable that the choice rule will remain the same. What we offer here, in any case, is an approximation to a choice rule which, in conjunction with minimal assumptions about the effects of experience on the attractiveness of the alternatives, makes it possible to simulate our data with considerable accuracy. The simulation is quantitative, and the fit to the data is quantitatively evaluated.

The attractiveness of each alternative (A or B) is given by the strength of its association with food, which is assumed to increase with reinforcement and to decrease with nonreinforcement. Change in associative strength is expressed by a linear equation (Bush \& Mosteller, 1951) in the familiar notation of Rescorla and Wagner (1972):

$$
\Delta \mathrm{V}_{\mathrm{A}}=\beta\left(\lambda-\mathrm{V}_{\mathrm{A}}\right)
$$

with $V_{A}$ representing the associative strength of alternative $A, \Delta V_{A}$ the change in strength produced by reinforcement or nonreinforcement, $\lambda$ the asymptotic associative strength, and $\beta$ the learning-rate parameter. The value of $\lambda$ is taken as 1 when there is reinforcement and as 0 when there is not, and provision is made in the model for the possibility that the value of $\beta$ is different for reinforcement and nonreinforcement $(\mathrm{U} \beta=$ incremental or up $\beta, D \beta=$ decremental or down $\beta$ ). For a reinforced choice, $\Delta V_{A}=U \beta \cdot\left(1-V_{A}\right)$; for an unreinforced choice, $\Delta V_{A}=D \beta \cdot\left(0-V_{A}\right)=-D \beta \cdot V_{A}$. To compare $V_{A}$ and $V_{B}$, we use a ratio rather than a difference score, primarily because normalization is so convenient. The relative strength of $A$ is designated as $r_{A}$ and is expressed by the equation

$$
r_{A}=V_{A} /\left(V_{A}+V_{B}\right) \text {, }
$$

where $V_{B}$ is the associative strength of $B$. A simple computer program evaluates $r$ on the basis of assumed initial values of $V_{A}$ and $V_{B}$ (note that $r_{B}=1-r_{A}$ ), dictates a choice, and then calculates the resulting changes in associative strength. When $A$ is the reinforced alternative on a given visit, $V_{A}$ alone is changed (incremented) if $A$ is chosen initially, and $V_{B}$ also is changed (decremented) if $B$ is chosen initially. Choice is dictated by any one of a variety of choice functions that may be incorporated in the model, that is, functions relating $\mathbf{P}_{\mathrm{A}}$, the probability of choosing $A$, to $r_{A}$. The accuracy of prediction from such a model depends, of course, on the way in which 
change in associative strength is calculated, and on the way in which differential associative strength is expressed, as well as on the choice function, but our strategy was first to vary only the choice function in the hope of finding one that would yield a reasonably good fit to the data.

The first trials were made with each of a set of seven exponential functions which differ only in the value of the exponent, $\mathrm{K}$, and are designated by those values. To generate the functions, we set $s=.5$ in the equation

$$
\mathrm{P}=.5+\mathrm{s}(2 \mathrm{r}-1)^{\mathrm{K}}
$$

for $\mathrm{r}>.5$, or

$$
P=.5-s(1-2 r)^{K}
$$

for $r<.5$. The functions are plotted in Figure 5 only for $r \geq .5$, because they are perfectly symmetrical for $r<.5$, with $P_{A}+P_{B}=1$ for all values of $K$. The $K=1$ function describes what may be thought of as matching, although it is not the reinforcement ratio that is matched but the ratio of associative strengths generated by the schedule of reinforcement. By the same token, $K=0$ describes maximizing, which is included only to provide a point of reference, since it obviously could not be expected to yield a good fit. The other functions were selected on intuitive grounds, $0<\mathrm{K}<1$ intermediate between matching and maximizing, and $\mathrm{K}>1$ deviating from matching in the opposite direction; there was no good reason to think that any of them might not yield a better fit than matching.

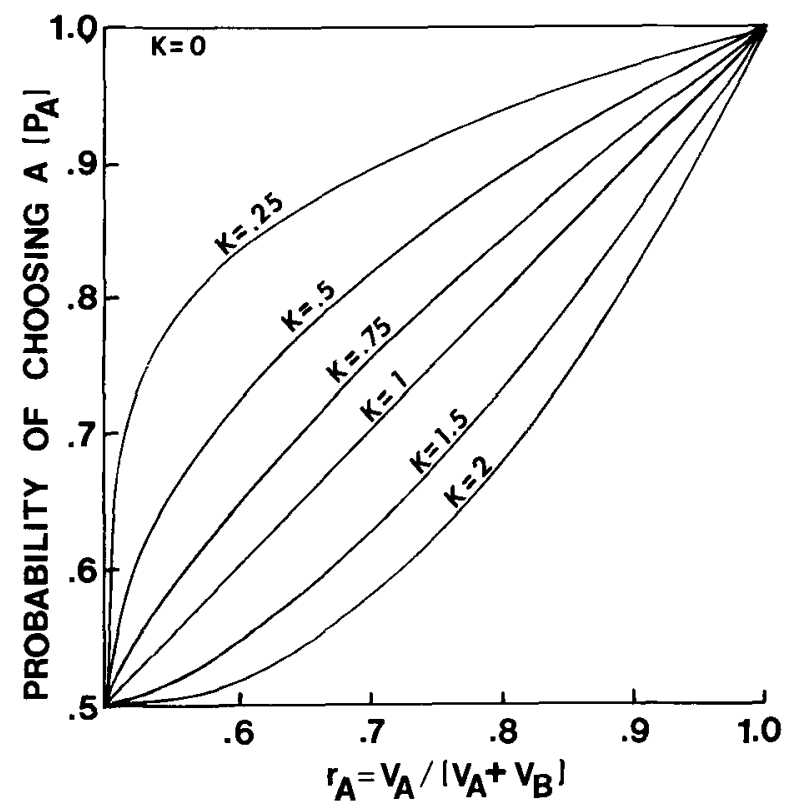

Figure 5. A set of choice functions, exponential in form, to which the matching $(K=1)$ and maximizing $(K=0)$ functions belong. $\mathbf{P}_{\mathrm{A}}=$ probability of choosing $A ; r_{A}=$ relative associative strength of $A$. Because the functions are symmetrical for values of $r_{A}<.5$, $P_{A}$ is plotted only for $r_{A} \geq .5$.
For each choice function, there were 100 simulations, each involving the training of 50 stat bees exactly as the real bees had been trained in each of the four experiments described above $(100: 0 \rightarrow 75: 25,50: 50,80: 20$, and $100: 0 \rightarrow 0: 100$ ). In the 100 simulations, 4 initial values of $\mathrm{V}_{\mathrm{A}}=\mathrm{V}_{\mathrm{B}}(.1, .2, .3$, and .4-all greater than 0 because of pretraining), 5 values of $\mathrm{U} \beta(.05, .1, .15, .2$, and .25), and 5 values of $D \beta$ (the same as for $U \beta$ ) were combined factorially. The use of such high values was suggested by the rapid learning observed in these and previous experiments. For each simulation, a root-meansquare (RMS) estimation of goodness of fit to the experimental results was computed on the basis of 61 values. For the 100:0 $\rightarrow$ 75:25 experiment, the means for the eight postcriterion (75:25) four-visit blocks were used. For each of the other three experiments, the values used were those for every visit except the first (the 34 values actually obtained in the 50:50 and 80:20 experiments, and 19 values taken from the smoothed acquisition and reversal curves in the 100:0 $\rightarrow 0: 100$ experiment). Blocked 75:25 data were used in the calculation because the subgroups trained with the two different 75:25 schedules were too small $(\mathrm{N}=4)$ to provide reliable visit-by-visit information on the effects of the schedules; in the simulations, each schedule was used for 25 stat bees and the results were combined. The first-visit points of the other three experiments were omitted because $\mathrm{P}_{\mathrm{A}}=.5$ at the outset for all of the choice functions to be evaluated; any deviation in the proportion of $A$ choices from .5 , either in the simulated data or (given the balanced design) in the real data, was assumed to represent sampling error. By the same token, the 100:0 and 0:100 curves were smoothed because none of the choice functions to be evaluated can predict the deviations, which are treated as error. For each choice function, then, a list of 100 RMS values was scanned to find the best fit (minimal RMS) and the parameters (initial $\mathrm{V}_{\mathrm{A}}=\mathrm{V}_{\mathrm{B}}, \mathrm{D} \beta$, and $\mathrm{U} \beta$ ) that yielded it.

The results for the seven functions are plotted in Figure 6 (the curve labeled $s=.5$ ). The curve shows systematic variation in minimal RMS with $\mathrm{K}$. For the maximizing function ( $K=0$ ), none of the fits is very good, and it makes little difference which parameters are used, the RMS values ranging only from .30 to .36 over the 100 sets. The fits to the blocked results of the 75:25 training are reasonably satisfactory-the maximizing rule may indeed yield matching of choice and reinforcement ratios when data pooled over trials are considered-but the deficiencies of the rule are evident as soon as individual trials are examined. There is, for example, one-trial acquisition in the simulated 100:0 training, and the simulated probability of choosing $A$ in the 50:50 training is always either 1 or 0 , depending on the reinforced alternative of the immediately preceding visit. With $\mathrm{K}=.25$, minimal RMS declines sharply, but there still is too much rewardfollowing. With $\mathrm{K}=.5$, minimal RMS declines further, and then it rises again as $K$ continues to increase. The best fits are obtained with relatively high $\beta$ values, but 
even so, the simulated terminal performance in the $100: 0$ and 0:100 training does not come up to the level attained by the real animals, which suggested to us that we might be underestimating the rate at which $\mathrm{P}_{\mathrm{A}}$ approaches 1 , especially for higher values of $r_{A}$.

Accordingly, we repeated the entire simulation process with the $\mathrm{K}>0$ functions steepened by setting $\mathrm{s}=.625$; the effect of this change on $\mathrm{K}=.75$ is shown in Figure 7 . Finally, simulations were done with four of the functions ( $\mathrm{K}=.5, \mathrm{~K}=.75, \mathrm{~K}=1$, and $\mathrm{K}=1.5$ ) steepened further by setting $\mathrm{s}=.833$; the effect on $\mathrm{K}=1$ also is shown in Figure 7. As Figure 6 indicates, increasing steepness substantially improved the fit. Analysis of variance based on the best simulations (minimal RMS) achieved with the four $K$ values $(.5, .75,1$, and 1.5$)$ studied at each of the three $s$ values $(.5, .625$, and .833) yields both a significant $K$ effect $[F(3,180)=2.94, p=.0346]$ and a significant $s$ effect $[F(2,120)=3.65, p=.0289]$, although the interaction of $\mathrm{K} \times \mathrm{s}$ is not significant $[\mathrm{F}(6,360)=$ $1.51, \mathrm{p} \geq .05]$. The two best fits are for $\mathrm{K}=.75, \mathrm{~s}=$ .625 (initial $\mathrm{V}_{\mathrm{A}}=\mathrm{V}_{\mathrm{B}}=.3, \mathrm{U} \beta=\mathrm{D} \beta=.25$ ) and for $\mathrm{K}=1, \mathrm{~s}=.833$ (same parameters); the former, marginally better, fit is shown along with the real data in Figures 1-4. In Figure 7, these two functions $(K=.75, \mathrm{~s}=$ .625 and $K=1, s=.833$ )-again only for $r \geq .5$-are compared with the maximizing function and with the original matching function $(K=1, \mathrm{~s}=.5$ ). An effort to define the "true" choice function more exactly with the data at hand probably is not warranted, because the RMS values for our best fits are so small as to suggest that we

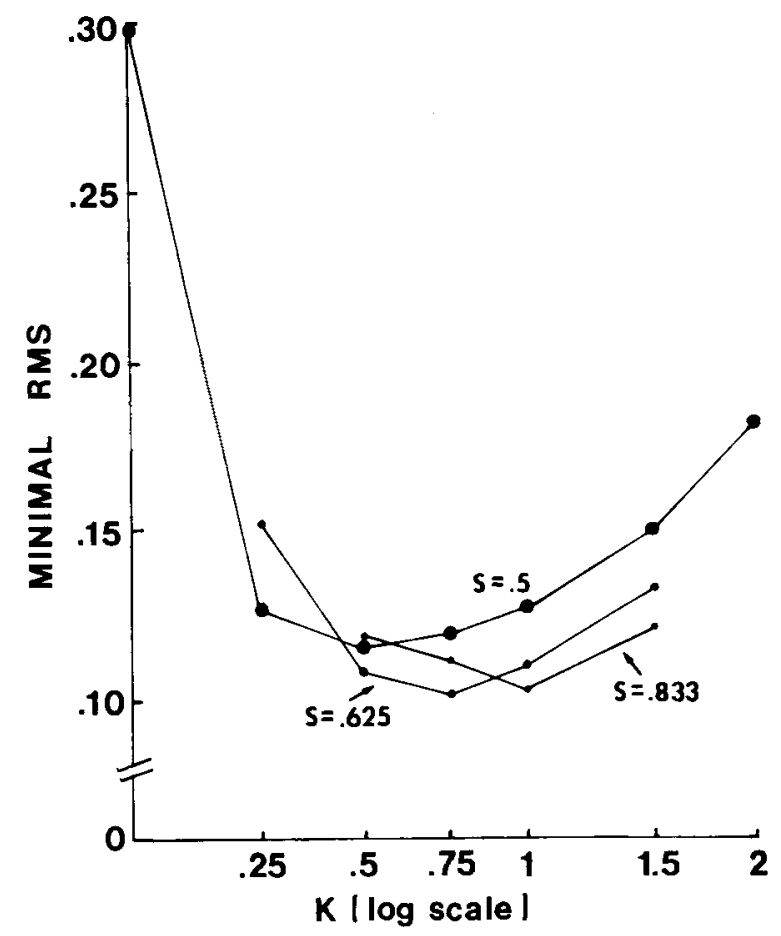

Figure 6. Minimal RMS deviations of simulated data from obtained data for choice functions varying in the value of the exponent, $K$, and in steepness, $s$.

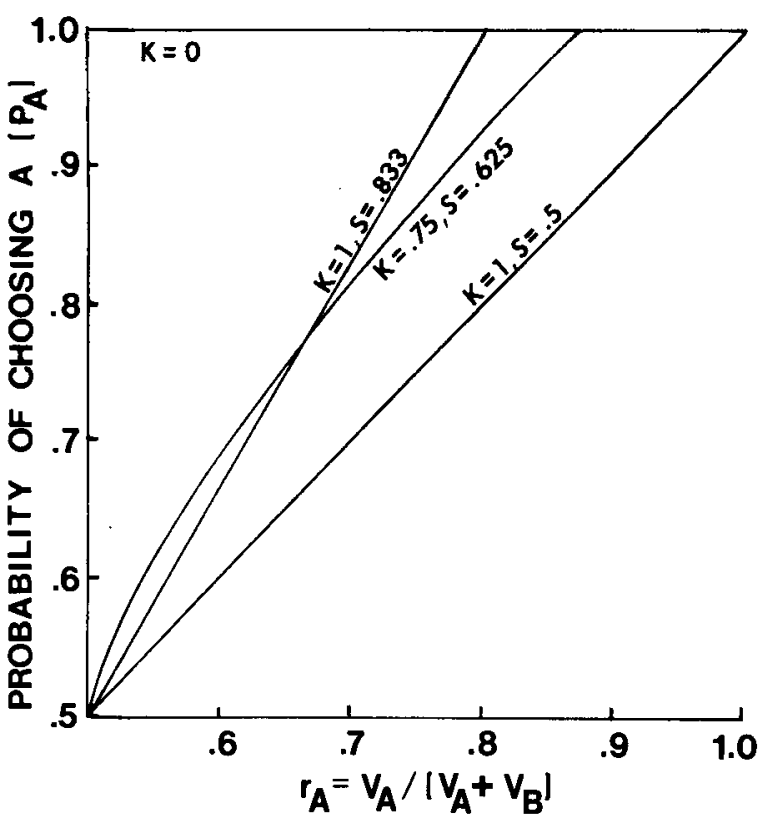

Figure 7. The two choice-functions that yielded the best fits $(K=$ $.75, s=.625$ and $K=1, s=.833$ ) compared with the maximizing function $(K=0)$ and the original matching function $(K=1$, $\mathbf{s}=\mathbf{. 5}$ ).

are already very close to the limit imposed by errors of measurement. A more promising approach would be to try to constrain the model further with more complex reinforcement schedules, using a larger number of subjects in each experiment to minimize errors of measurement.

It may be well to note that at the suggestion of reviewers we simulated Sigurdson's (1981) probability experiment, which we had not bothered to do before because blocked data did not seem to us to constrain the model sufficiently. The data reported by Sigurdson were for 6 bees trained to discriminate between two colors in 20 blocks of 40 trials (10 per visit) with a reinforcement ratio of $70: 30$ in the first 10 blocks and of 30:70 in the last 10 blocks. The 70:30 training was preceded by four 10-trial visits of 100:0 training, the unpublished data of which were provided by Sigurdson. Because the amount of reward was relatively small in Sigurdson's experiment, we did 10 simulations, each with $K=.75, \mathrm{~s}=.625, \mathrm{~V}_{\mathrm{A}}=\mathrm{V}_{\mathrm{B}}=.3$, and $\mathrm{U} \beta=\mathrm{D} \beta=.25$, but with $\lambda$ varying from .1 to 1 in steps of .1 . For the 70:30 $\rightarrow 30: 70$ data, the results for all values of $\lambda$ were equally good: The RMS deviations of the means for 35 stat bees from the means for the real bees varied unsystematically from .065 to .071 . Where $\lambda$ did seem to make a substantial difference was in the initial 100:0 training, the simulated rate of acquisition being too slow at the lowest values and too rapid at the highest values-the best fit was for $\lambda=.3$, RMS deviation $=.025$-but wider variation in magnitude of reward under a range of experimental conditions will be required before its role can adequately be assessed.

The good fits already obtained suggest not only that we are approaching a definition of the choice function for 
honeybees, but that the other assumptions on which the simulations were based are approximately correct, if only in a rather global sense, and that some effort to refine them is warranted. Needless to say, the associative features of the model with which we have been working are vastly oversimplified. For example, we have ignored entirely the possibility that position acquires associative control of responding, although under conditions like ours honeybees do, in fact, come to choose in terms of position when it is differentially reinforced (Klosterhalfen, Fischer, \& Bitterman, 1978). Clearly, the computation of $r$ on each occasion of choice should take account both of properties common to the alternatives (such as those of the gray target itself) and of nondifferentially reinforced differences in their properties (such as their positions). To do so, of course, would require explicit assumptions about the processes involved in compound conditioning and in stimulus compounding, which may be almost as complicated in honeybees as in vertebrates (Couvillon \& Bitterman, 1982; Couvillon, Klosterhalfen, \& Bitterman, 1983). We shall want, certainly, to explore the usefulness of the concept of shared associative strength (Rescorla \& Wagner, 1972) in experiments more suitable for the purpose than the present ones, which did not require it. Other experiments with honeybees (Couvillon \& Bitterman, 1984) suggest that unreinforced experience with a given alternative may not, under certain circumstances, simply reduce the strength of its association with food, but may generate a competing, frustration-like process that may itself be conditioned, and even counterconditioned (cf. Daly \& Daly, 1982). There is no independent evidence yet for conditioned frustration in bees-the overlearning extinction effect and the negative-contrast effect we have found can be understood in terms of unconditioned frustration alone-but it is conceivable that the high down $\beta$ required by our data reflects the operation of such a process. However complex the assumptions that may be suggested by the results of further experiments, the most straightforward way to establish their validity is by quantitative simulation, and even such modest achievements as are reported here promise eventual success in that enterprise.

\section{REFERENCES}

Bitterman, M. E. (1975). The comparative analysis of learning. Science, 188, 699-709.
Bullock, D. H., \& Bitterman, M. E. (1962). Probability-matching in the pigeon. American Journal of Psychology, 75, 634-639.

BUSH, R. R., \& MosTELLER, F. (1951). A mathematical model for simple learning. Psychological Review, 58, 313-323.

Couvillon, P. A., \& Bitterman, M. E. (1980). Some phenomena of associative learning in honeybees. Journal of Comparative \& Physiological Psychology, 94, 878-885.

Couvillon, P. A. \& Bitterman, M. E. (1982). Compound conditioning in honeybees. Journal of Comparative \& Physiological Psychology, 96, 192-199.

Couvillon, P. A., \& Bitterman, M. E. (1984). The overlearningextinction effect and successive negative contrast in honeybees (Apis mellifera). Journal of Comparative Psychology, 98, 100-109.

Couvillon, P. A., Klosterhalfen, S., \& Bitterman, M. E. (1983). Analysis of overshadowing in honeybees. Journal of Comparative Psychology, 97, 154-166.

DALY, H. B., \& DALY, J. T. (1982). A mathematical model of reward and aversive nonreward: Its application in over 30 appetitive learning situations. Journal of Experimental Psychology: General, 111, 441-480.

HeRRNSTEIN, R. J. (1970). On the law of effect. Journal of the Experimental Analysis of Behavior, 13, 243-266.

HERRNSTEIN, R. J. (1982). Melioration as behavioral dynamism. In M. L. Common, R. J. Herrnstein, \& H. Rachlin (Eds.), Quantitative analysis of behavior: Matching and maximizing accounts (pp. 433458). Cambridge, MA: Ballinger.

Kamil, A. C., Peters, J., \& Lindstrom, F. J. (1982). An ecological perspective on the study of allocation behavior. In M. L. Common, R. J. Herrnstein, \& H. Rachlin (Eds.), Quantitative analysis of behavior: Matching and maximizing accounts (pp. 189-203). Cambridge, MA: Ballinger.

Klosterhalfen, S., Fischer, W., \& Bitterman, M. E. (1978). Modification of attention in honeybees. Science, 201, 1241-1243.

LoNGo, N. (1964). Probability-learning and habit-reversal in the cockroach. American Journal of Psychology, 77, 49-51.

MackInTOSH, N. J. (1974). The psychology of animal leaming. London: Academic Press.

Menzel, R., \& Bitterman, M. E. (1983). Learning by honeybees in an unnatural situation. In F. Huber \& H. Markl (Eds.), Neuroethology and behavioral physiology (pp. 206-215). Berlin: Springer.

RESCORLA, R. A., \& WAGNER, A. R. (1972). A theory of classical conditioning: Variations in the effectiveness of reinforcement and nonreinforcement. In A. H. Black \& W. F. Prokasy (Eds.), Classical conditioning II: Current research and theory (pp. 64-99). New York: Appleton-Century-Crofts.

SHIMP, C. P. (1982). Reinforcement and the local organization of behavior. In M. L. Common, R. J. Herrnstein, \& H. Rachlin (Eds.), Quantitative analysis of behavior: Matching and maximizing accounts (pp. 111-130). Cambridge, MA: Ballinger.

Sjgurdson, J. E. (1981). Automated discrete-trials techniques of appetitive conditioning in honey bees. Behavior Research Methods \& Instrumentation, 13, 1-10.

Sutherland, N. S., \& Mackintosh, N. J. (1971). Mechanisms of animal discrimination learning. New York: Academic Press.

(Manuscript received November 30, 1984; revision accepted for publication July 22,1985 .) 